\title{
Exploración de la percepción social de la ciencia y la tecnología de ingenieros químicos colombianos y sus docentes
}

\section{Exploration of the science and technology social perception of Colombian chemical engineers and their teachers}

\author{
Cristián Vargas-Ordóñez \\ Maestría en Ciencia, Tecnología y Sociedad, Universidad Nacional de Quilmes, Argentina. \\ Correo electrónico: cevo8507@gmail.com
}

Recibido: 11 noviembre, 2017. Aceptado: 21 octubre, 2018. Versión final: 28 enero, 2019.

\begin{abstract}
Resumen
Esta investigación busca explorar la percepción social de la ciencia y la tecnología (PSCyT) de estudiantes, profesionales, docentes y directivos de la carrera de Ingeniería Química de universidades colombianas, bajo la visión del ingeniero químico como un agente activo dentro de la ciudadanía. De esta manera, la hipótesis de trabajo consiste en que la percepción de los ingenieros químicos hacia la ciencia y la tecnología es similar a la de los ciudadanos no ingenieros químicos. Así, como base conceptual se tuvieron en cuenta las representaciones sociales (RS) de Moscovici (actitudes, intereses y campos de representación), así como las RS de Abric (núcleo y periferia), con el fin de brindar soporte a las representaciones sociales de la ciencia y la tecnología (RSCyT) y, por ende, a la percepción social de la ciencia y la tecnología. Metodológicamente, se configuró una encuesta con preguntas abiertas, de listados libres, y cerradas, con opción múltiple, basadas en preguntas adaptadas de tres estudios previos. Entre los resultados se encontró que los encuestados poseen un concepto más unificado de ciencia desde el enfoque neopositivista, mientras que tienen un concepto más disperso, pero con actitudes positivas, frente a la definición de tecnología. Asimismo, esta población se autopercibe cercana a la solución de problemáticas ambientales, pero no así hacia aquellas de orden social. Como conclusión, la PSCyT de los sujetos encuestados es similar a aquella encontrada en los ciudadanos no ingenieros químicos en cuanto a las creencias e ideas sobre los conceptos de ciencia y tecnología (CyT), comunicación y consulta de temas científicos y tecnológicos. Por su parte, esta percepción se identifica diferente con relación a la asistencia a actividades de CyT, sobre cómo son valoradas y sus formas de participación como ciudadanos. Este estudio se convierte en una valiosa oportunidad para impulsar nuevas investigaciones que ayuden a determinar el papel del conocimiento obtenido desde la academia en la concepción de estos factores, así como para mejorar los currículos propuestos por las diversas instituciones de educación superior (IES).
\end{abstract}

Palabras clave: ingeniería química; percepción social de la ciencia y la tecnología; participación ciudadana; representaciones sociales.

\footnotetext{
Abstract

The present research work explores the Science and Technology's Social Perception (STSP) of Chemical Engineering (CE) students, professionals, teachers and directives from Colombian universities. Under a constructivism focus, this research visualizes the chemical engineer as an active agent in society. Therefore, the hypothesis considers that chemical engineers' science and technology perception is similar to that of the citizens who are not part of this community. Moscovici's theory of Social Representation (RS) (attitudes, interests and representations fields) and 
Abric's model of this human process (core and periphery) are the conceptual basis of Science and Technology Social Representations (STSR) and thus, Science and Technology Social Perception. A survey consists of opened-ended question like free listing and close-ended questions with multiple choice, which were adapted from three previous studies. Results found that participants have a neo-positivist unified concept of science while they have a disperse concept of technology but with positive attitudes. Also, this community perceives itself that is close to the solution of environmental problems but far from social problems. In conclusion, the STSP in the participants is similar to the one encounter in non-chemical engineers about beliefs and ideas related to the concepts of Science and Technology (S\&T) and communication and consult of scientific and technological topics. However, this perception is different from the one of non-chemical engineers regarding the attendance to S\&T activities, valuation of these processes and the kind of citizen participation they have. Therefore, this study becomes an opportunity to impulse new researches to improve the suggested curriculums for the different universities.

Keywords: science and technology social perception; social representations; citizen participation; chemical engineering.

\section{Introducción}

Los ingenieros químicos, como parte de la ciudadanía, son parte importante en la elaboración de políticas públicas de ciencia y tecnología (CyT, en adelante) que sean incluyentes, estén aterrizadas a la realidad del país y permitan el desarrollo económico y social. Directa o indirectamente, todos los ciudadanos en el mundo son parte activa de la toma de decisiones en este tipo de políticas públicas y más aún luego de los resultados científicos de la Segunda Guerra Mundial, con el Proyecto Manhattan y la bomba atómica [1]. En ese orden de ideas, la ciencia y la tecnología se constituyen en poderosas herramientas de interacción entre el ser humano y sus entornos ambiental, social o político inmediatos, las cuales sustentan las ideas de desarrollo económico y social del sistema económico que predomina en la actualidad. En el caso de Colombia, entre las proyecciones de desarrollo económico y social en el país, este se asocia al crecimiento de la industria y en particular al de las industrias energética, manufacturera y de servicios [2], entendidas como consecuencia de procesos científicos y tecnológicos. Por tanto, los ingenieros químicos se convierten en actores importantes dentro de este fenómeno, para lo cual es relevante identificar cómo conciben la ciencia y la tecnología como comunidad. El trabajo de investigación que se expone apunta, precisamente, a explorar la percepción social de la ciencia y la tecnología (PSCyT, en adelante) en ingenieros químicos colombianos y sus docentes.

La PSCyT nace del concepto de representaciones sociales (RS, en adelante), el cual ha sido definido significativamente en los trabajos desarrollados por Serge Moscovici y Jean Claude Abric. Según Moscovici, las RS son procesos psicológicos en constante cambio a través de los cuales el ser humano entiende la realidad física y social por medio de la comunicación y la interacción con el entorno [3]. Asimismo, se basa en las actitudes (acciones que se entretejen alrededor de un tema en común), la información (grado de conocimiento que tiene un grupo con relación a un objeto social) y el campo de representación social o imagen (modelo construido socialmente sobre un elemento puntual del objeto social). Por otro lado, Abric las define, análogamente a las percepciones sociales (PS, en adelante), como una forma funcional de entender $\mathrm{e}$ interpretar la realidad desde el propio sistema de referencias, el cual rige la interacción entre los individuos y su entorno físico y social y establece los comportamientos y prácticas de estos [4]. De esta manera, estas formas de ver el mundo son divididas en nucleares (sistemas centrales de valores culturales que son estables, conducen la conducta y dan sentido a los hechos) y periféricas (formas externas al núcleo que permiten el acceso a este a través del anclaje/concreción de la $\mathrm{RS}$ a la realidad, la regulación/adaptación de la RS al contexto y la defensa/ponderación ante la nueva información).

El efecto de las RS en los estudios de PSCyT se remonta a sus orígenes. Como resultado de la propuesta de utilizar a la ciencia y la tecnología como ejes de desarrollo económico y social, luego de las notorias consecuencias del Proyecto Manhattan en la sociedad, en los años cincuenta surgieron encuestas de opinión en torno a la cantidad de conocimiento científico que el público tenía. No obstante, estas indagaciones se basaron en el modelo deficitario cognitivo (la ciencia y los científicos como un punto que debe alcanzarse por el público "lego"), las cuales consideraron que un sujeto, mientras más cercano a la ciencia sea, tiene mayores probabilidades de evaluarla positivamente [5]. En los años setenta, esta concepción se fracturó debido a las críticas sobre la exclusión que este modelo forja. De esta forma, nacieron los estudios de PSCyT como encuestas que medían, en un principio, el grado de alfabetización científica 
(Scientific Literacy) o de comprensión pública de la ciencia (Public Understanding of Science). Principalmente, los estudios de comprensión pública de la ciencia incorporaron las actitudes, los intereses y los campos de representación de la ciudadanía frente a la ciencia como categorías del análisis por realizar y generaron fuertes influencias en las políticas de CyT, debido a los resultados hallados. De esta forma, diversos países incorporaron estos estudios como una práctica regular que genera unos insumos para la formulación y reformulación de políticas públicas aterrizadas a los contextos donde son elaboradas. No obstante, a medida que los estudios fueron más recurrentes y los resultados obtenidos permitieron comparar los diferentes abordajes teóricos que los países han utilizado, las regiones comenzaron a generar marcos metodológicos y conceptuales de referencia para facilitar dicha comparación, como el Proyecto iberoamericano de indicadores de percepción pública, cultura científica y participación ciudadana, por ejemplo. De esta forma, además de las categorías anteriores, estos estudios incorporaron a la participación ciudadana como un nuevo componente para evaluar, al considerar a la ciudadanía como precursora, promotora y contenedora de la cultura científica.

De esta manera, identificar la PSCyT de un grupo específico ayuda a brindar una perspectiva particular de este fenómeno social, lo cual se traduce en la validación conceptual de los estudios que previamente se han realizado y en un uso diferente de este tipo de estudios. Así, la hipótesis de trabajo de la investigación se centró en considerar que los ingenieros químicos no tienen una PSCyT diferente a la ciudadanía general, dado que hace parte de esta en su diario vivir. De esta forma, se sitúa a esta investigación desde un enfoque analítico, el cual utiliza como base conceptual y metodológica a la psicología social y como recursos de comparación y análisis de los resultados al Proyecto iberoamericano de percepción pública, cultura científica y participación ciudadana [6], la III Encuesta de percepción pública de la ciencia y la tecnología, Colombia 2012 [7] y a Los Estudiantes y la ciencia. Encuesta a jóvenes iberoamericanos [8]. Asimismo, el presente documento muestra los resultados del trabajo Exploración de la percepción social de la ciencia y la tecnología en ingenieros químicos colombianos y sus docentes [9].

\section{Metodología}

El estudio consistió en indagar, a través de un cuestionario virtual de preguntas abiertas y cerradas, las categorías de análisis anteriormente expuestas. Para ello, se realizó un muestreo no probabilístico por bola de nieve a 318 personas, entre estudiantes, ingenieros químicos graduados, docentes y directivos de esta carrera en el país. Se elaboraron preguntas de opción múltiple con única respuesta, abierta tipo listado libre, opción múltiple con múltiple respuesta y preguntas de respuestas escalares de 3 niveles. Asimismo, se validó la encuesta a través de una validación por expertos y una prueba piloto a 23 participantes ingenieros químicos profesionales y docentes, de la cual se realizó la reformulación de la pregunta 406 y la distribución espacial de la encuesta. El análisis de la información se realizó conforme a la naturaleza cuantitativa o cualitativa de los datos.

En cuanto al análisis cuantitativo, se desarrolló un análisis descriptivo y de diferencia de medias y correlaciones a través de SPSS v.22 [10] y Microsoft Excel. Se distinguieron las variables como categóricas o continuas. Entre las variables categóricas se consideraron el sexo, actividad, universidad-estudiantes, semestre, universidad-graduados, actividad principal y universidad docente, con el fin de analizar las preguntas medios consulta, medios publica, 303 y 40-406. A su vez, estas variables se clasificaron en significativas $(p<0,005)$ y no significativas ( $p>0,005)$, según el análisis estadístico correspondiente. Con respecto a las variables continuas se consideraron la edad y el año de graduación; para el análisis de las preguntas, medios consulta y medios publica, a través de un ANOVA (pi<0,005) de un factor y Pruebas de Scheffe ( $\mathrm{pb}<0,005)$, como pruebas post hoc. Finalmente, las preguntas 303, 401C y 406-2 o menos opciones de respuestas- fueron analizadas por diferencia de medias de muestras independientes, las cuales utilizaron la media, la varianza, el número de casos y la significancia (pi<0,05; pb<0,005) como variables por considerar, por medio de la Prueba de Levene de Calidad de Varianzas.

Por su parte, el análisis cualitativo de los listados se realizó en tres etapas: análisis de frecuencias, análisis de nodos y grafos de asociación o de redes y análisis de asociación. El análisis de frecuencias se realizó por Microsoft Excel 2016, para determinar la frecuencia global de las palabras. Por otro lado, el análisis de nodos y grafos de asociación se realizó a través del programa libre Gephi 0.8 .2 beta. Finalmente, el análisis de asociación se realizó por medio del programa de minería de datos Weka 3, para encontrar las parejas de respuestas e identificar aquellas más frecuentes a través de los parámetros de soporte y confianza. De esta manera, se decidió que solamente se tendrían en cuenta los resultados con confianzas (cantidad de veces por fracción que al aparecer una palabra $\mathrm{X}$, aparece la palabra $\mathrm{Y}$ ) y soportes (total de veces que aparece una pareja de palabras en un conjunto de datos, XUY) mayores a $0,1 \mathrm{y}$ 0,02 , respectivamente. 


\section{Resultados}

\subsection{Resultados sociodemográficos}

De los 318 participantes, el 66,0\% fueron estudiantes de ingeniería química; el 25,5\%, ingenieros químicos graduados, y el 8,5\%, docentes y directivos de la carrera de ingeniería química. Asimismo, el estudio contó con participantes entre los 15 y 65 años de edad, lo cual muestra una edad promedio total de 23 años ( $\mathrm{SD}=7$ años). Igualmente, no se encontraron diferencias significativas en la edad por sexo, entre grupos por actividad o en la muestra total (hombres $=24$ años; $y$ mujeres $=23$ años). En cuanto a la universidad a la que pertenecen los encuestados, se observó que la Universidad Nacional de Colombia - Sede Bogotá fue aquella con mayor participación de estudiantes $(36,7 \%)$; la Fundación Universidad de América, con mayoría de los ingenieros químicos graduados $(42,0 \%)$, y la Universidad de la Sabana, respectivamente, con los docentes y directivos $(44,4 \%)$.

\subsection{Creencias e ideas}

En cuanto a ciencia, los encuestados la perciben como un fenómeno teórico y experimental que se elabora en el laboratorio y donde priman el conocimiento y la investigación experimental (Figura 1). De esta manera, existe un núcleo representativo claro (Investigación) que evidencia un consenso sobre este tema, lo cual parece estar relacionado con el concepto heredado de la ciencia [11]. Por su parte, la tecnología (Figura 2) se configura como un concepto menos compacto que el anterior, lo que evidencia la ausencia de un núcleo representativo claro y un menor consenso al respecto. En ese orden de ideas, se le identifica como un conjunto de herramientas (concepción artefactual de la tecnología) que están asociadas al desarrollo económico y social (determinismo tecnológico), y que permiten transmitir información y conocimiento. De tal forma, este concepto se enmarca dentro de la concepción artefactual de la tecnología (en la que esta se considera como un objeto) [11], el determinismo tecnológico (la tecnología define cómo actuará la sociedad) [12] y la sociedad del conocimiento [13]. Por tanto, esta concepción se basa en la definición restringida de tecnología propuesta por Pacey [14], debido a que en esta "solo se hace referencia al aspecto técnico, conocimiento, destreza y técnica, herramientas, máquinas o recursos" [11], la cual es la característica principal de las concepciones intelectualista y artefactual de la tecnología.

Por otra parte, los encuestados conciben los productores de CyT a instituciones tales como las Universidades o el Instituto Colombiano del Petróleo como nodos de asociación principales. Sin embargo, esta misma población menciona más frecuentemente a la Universidades $(43,8 \%) \quad y \quad$ al Departamento Administrativo de Ciencia y Tecnología (Colciencias) $(24,4 \%)$ como las instituciones más cercanas a este proceso. En general, para los participantes, la CyT colombianas se producen perceptivamente de forma más cercana a acciones generadas por los grupos de investigación de las IES. Asimismo, se vincula esta producción a algunos centros de investigación y a programas especiales de ciencia, tecnología e innovación (Figura 3). En tanto los divulgadores de CyT son asociados con mayor frecuencia con Colciencias (29,0 $\%)$ y las universidades $(20,1 \%)$ y las revistas científicas. No obstante, como nodos de asociación, se consideran a las universidades y las revistas científicas, dadas las funciones no divulgadoras que ejerce Colciencias. De lo anterior, es posible establecer que los resultados obtenidos dependen de qué se divulgue y quién lo haga, lo cual se relaciona con cuán cercano está el encuestado, hacia la educación formal recibida en las instituciones de educación superior (IES) (Figura 4).

\subsection{Acciones}

En esta categoría, según los encuestados, entre los medios de comunicación usados para consultar o recibir información de CyT se concibe al internet como aquel medio más importante $(68,9 \%)$ [15], y resulta interesante que las redes sociales sean las más utilizadas para divulgar por los estudiantes [16]. Sin embargo, una de las limitantes del instrumento de evaluación utilizado consistió en concebir únicamente la transmisión de información sin llegar a identificar si esta se apropia y transforma en conocimiento. Por otro lado, los medios de comunicación usados para publicar o comunicar información de CyT serán diferentes en función del grupo que se mencione. De esta forma, los estudiantes utilizan redes sociales para la comunicación científica y tecnológica $(38,1 \%)$, mientras que los profesionales o docentes/directivos consideran el uso de revistas especializadas. Esto podría asociarse al tipo de información que se divulga (formal o informal) y al papel, experticia y rol de quien comunica este tipo de información [17].

Por otra parte, de acuerdo con los resultados obtenidos, los encuestados manifiestan acudir en mayor medida a bibliotecas $(79,2 \%)$, conferencias $(63,5 \%)$, museos de CyT $(35,8 \%)$ y semanas de la ciencia $(20,4 \%)$; con respecto a los resultados hallados en la encuesta colombiana $(38,00 \%, 22,81 \%, 19,74 \%$ y $11,34 \%$, respectivamente) o la encuesta a estudiantes de bachillerato (museos: $14 \%$ y semanas ciencia: $10 \%$ ), luego de graduarse, este interés parece disminuir. Por tanto, la pertenencia directa a la universidad parece 
aumentar el interés en asistir a las bibliotecas. Tal vez, la necesidad de nuevo conocimiento, ya sea para continuar formándose o para investigación, sea el motor que impulsa la asistencia a este lugar. No obstante, asistir a conferencias o semanas de la ciencia se relaciona más con actividades del contexto académico, dado que los estudiantes y los docentes/directivos son quienes más afirman participar. Es decir, asistir a estos eventos podría relacionarse con la necesidad y gusto por información reciente que, posiblemente, pueda solucionar dudas o entregue adelantos de un tema de interés en particular dentro del ámbito académico. Del mismo modo, la importancia de esta forma de comunicarse sobre la CyT de la época se remonta a las conferencias científicas como vehículos de comunicación efectivos entre los científicos desde el siglo XVII [18]. Esta estrategia fue apropiada por las IES, lo cual influyó en los diferentes actores (docentes y directivos, especialmente) involucrados en estas, lo que se ve reflejado en los resultados obtenidos en la presente investigación (dictar conferencia: $66,7 \%$ ).

\subsection{Valores}

A continuación, se relacionan los resultados obtenidos para esta categoría según la subcategoría analizada:

\subsubsection{Interés en ciencia y tecnología}

Inicialmente, se observó que los ingenieros químicos, independientemente de la actividad que desarrollen (estudiantes, graduados, docentes o directivos), tienen un interés más cercano los productos $(74,2 \%)$, la aplicación de la ciencia $(67,0 \%)$, procesos industriales $(63,5 \%)$ y la invención o prueba de cosas nuevas $(57,5 \%)$. Por otro lado, su interés por el uso de herramientas electrónicas $(13,2 \%)$, TIC (18,6\%), la construcción de cosas $(22,3$ $\%)$ y generar ideas de carácter técnico (18,9\%), enfocarse en problemas sociales $(23,9 \%)$ o hacia las ciencias básicas $(48,4 \%)$ no son consideradas de su inmediato interés. Es decir, los encuestados tienen intereses en la ciencia ligados a los conocimientos brindados desde su formación como ingenieros químicos, más no por aquellos que podrían considerarse como externos a esta disciplina. Al parecer, los ingenieros químicos colombianos poseen una visión limitada de ellos mismos y no se consideran desde el sentido amplio visibilizado por la semántica, en la que se le supone como un agente que utiliza su ingenio y creatividad para resolver los problemas de la cotidianidad, incluyendo los problemas de orden social [19].

\subsubsection{Importancia de la ciencia y la tecnología}

Para el 86,9 \% de los encuestados la CyT son muy importantes para la innovación en las empresas, la utilización adecuada de los recursos naturales, el desarrollo económico nacional, el conocimiento y la preservación del entorno y el medioambiente, el cuidado de la salud y la prevención de enfermedades, y la comprensión del mundo. Implícitamente, la ciencia y la tecnología son vistas de suma importancia para el desarrollo y mantenimiento de la industria y el sistema económico actual [20] -lo cual estaría asociado con la percepción positiva de la CyT, observado en los anteriores apartados-. A su vez, los participantes dejan de lado la importancia de estos procesos como medios para relacionarse consigo mismo o los demás, lo cual podría relacionarse con la percepción de que la CyT asociada con sus conocimientos y metodologías son más cercanas a las ciencias naturales que a las ciencias sociales o humanas. Por su parte, en la Tabla 1 se muestra la comparación de algunos de los resultados obtenidos con aquellos de la encuesta colombiana [7] en cuanto a la importancia de la CyT para crear opiniones políticas, la toma de decisiones como consumidor, su relevancia para el campo de la salud o en la preservación del medioambiente.

Tabla 1. Comparación útiles y muy útiles.

\begin{tabular}{|l|c|c|}
\hline \multicolumn{1}{|c|}{ Categorías } & $\begin{array}{c}\text { Resultados } \\
(\boldsymbol{\%})\end{array}$ & $\begin{array}{c}\text { Encuesta } \\
\text { colombiana }(\boldsymbol{\%})\end{array}$ \\
\hline $\begin{array}{l}\text { Comprensión del } \\
\text { mundo }\end{array}$ & 85,0 & 76,6 \\
\hline Salud & 94,2 & 86,7 \\
\hline $\begin{array}{l}\text { Conservación } \\
\text { entorno /ambiente }\end{array}$ & 93,9 & 74,8 \\
\hline $\begin{array}{l}\text { Decisiones } \\
\text { consumidor }\end{array}$ & 64,5 & 70,7 \\
\hline $\begin{array}{l}\text { Opiniones } \\
\text { políticas }\end{array}$ & 42,5 & 51,3 \\
\hline
\end{tabular}

Fuente: elaboración propia.

\subsubsection{Usos de la ciencia y la tecnología}

De manera global, los encuestados consideraron que la CyT permiten en mayor medida entender el entorno $(85,7$ $\%)$, conocer la verdad $(76,5 \%)$ o solucionar problemas sociales $(86,7 \%)$, en comparación con ganar dinero $(61,5$ $\%$ ) o tener poder $(40,1 \%)$. En pocas palabras, la CyT son vistas como agentes alejados que influyen de forma altruista al entorno de los encuestados y que no interactúan en su diario vivir ni son útiles para alcanzar sus expectativas personales [21]. No obstante, los docentes y directivos son quienes manifiestan en menor medida que la CyT son útiles para ganar dinero $(44,4 \%)$ u obtener poder $(29,6 \%)$ con relación al resto de encuestados. 
Así, esto último podría asociarse con una visión utópica de la $C y T$ (en la que estos dos procesos son considerados únicamente como agentes generadores de nuevo conocimiento y equipos), una visión altruista desde los mismos docentes y directivos (en la que ganar dinero $\mathrm{u}$ obtener poder son intereses de otras poblaciones diferentes a ellos, ya sea por un statu quo que es necesario mantener o porque realmente así lo consideran), o una forma de rebeldía a través de la cual se resisten a que la CyT deban ser utilizadas para tales fines.

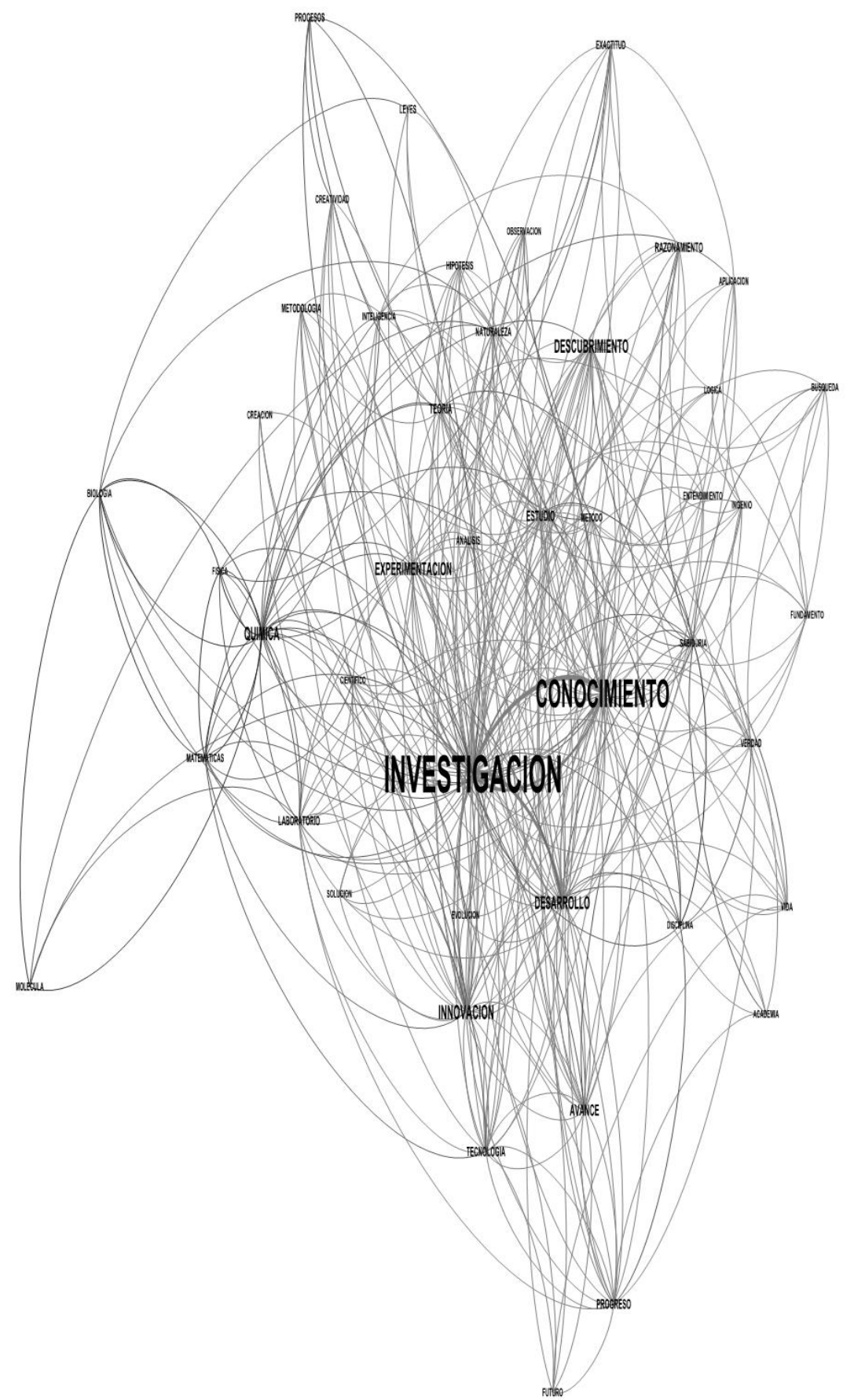

Figura 1. Análisis social de redes para la palabra 'ciencia', según el grupo poblacional encuestado. Fuente: elaboración propia. 


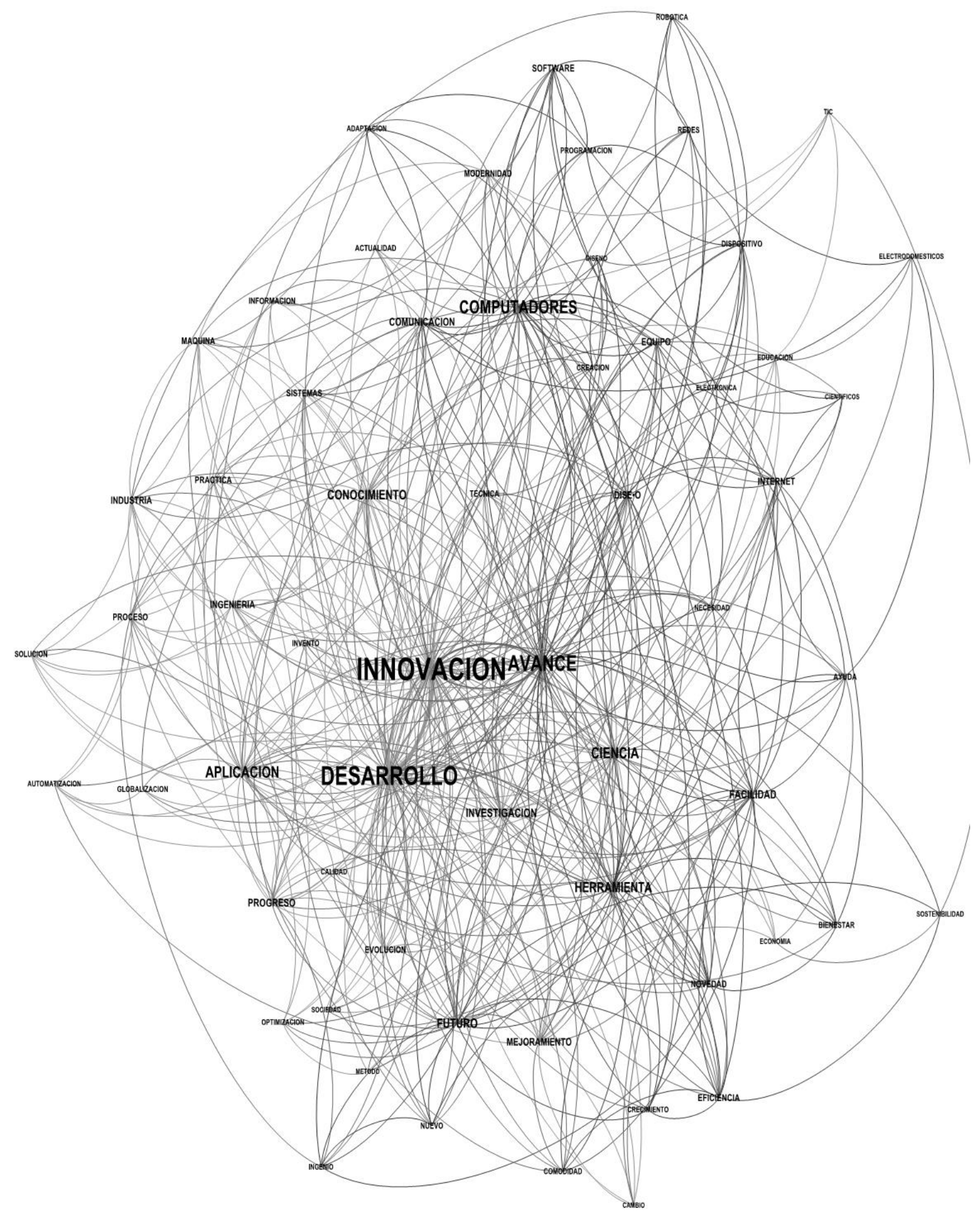

Figura 2. Análisis social de redes para la palabra 'tecnología', según el grupo poblacional encuestado. Fuente: elaboración propia. 


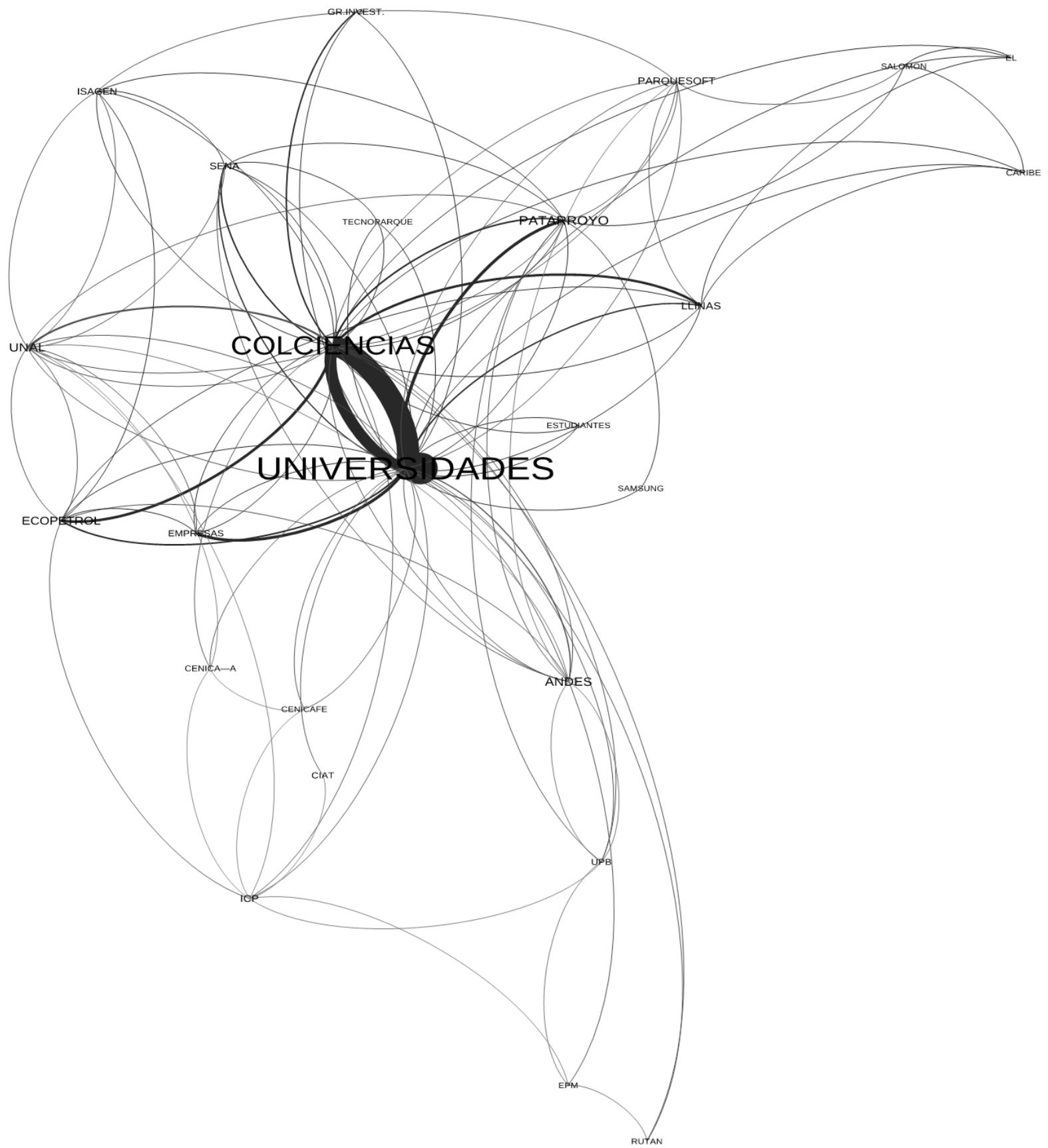

Figura 3. Análisis social de redes para identificar organizaciones, instituciones o personas productoras de ciencia y tecnología en Colombia. Fuente: elaboración propia. 


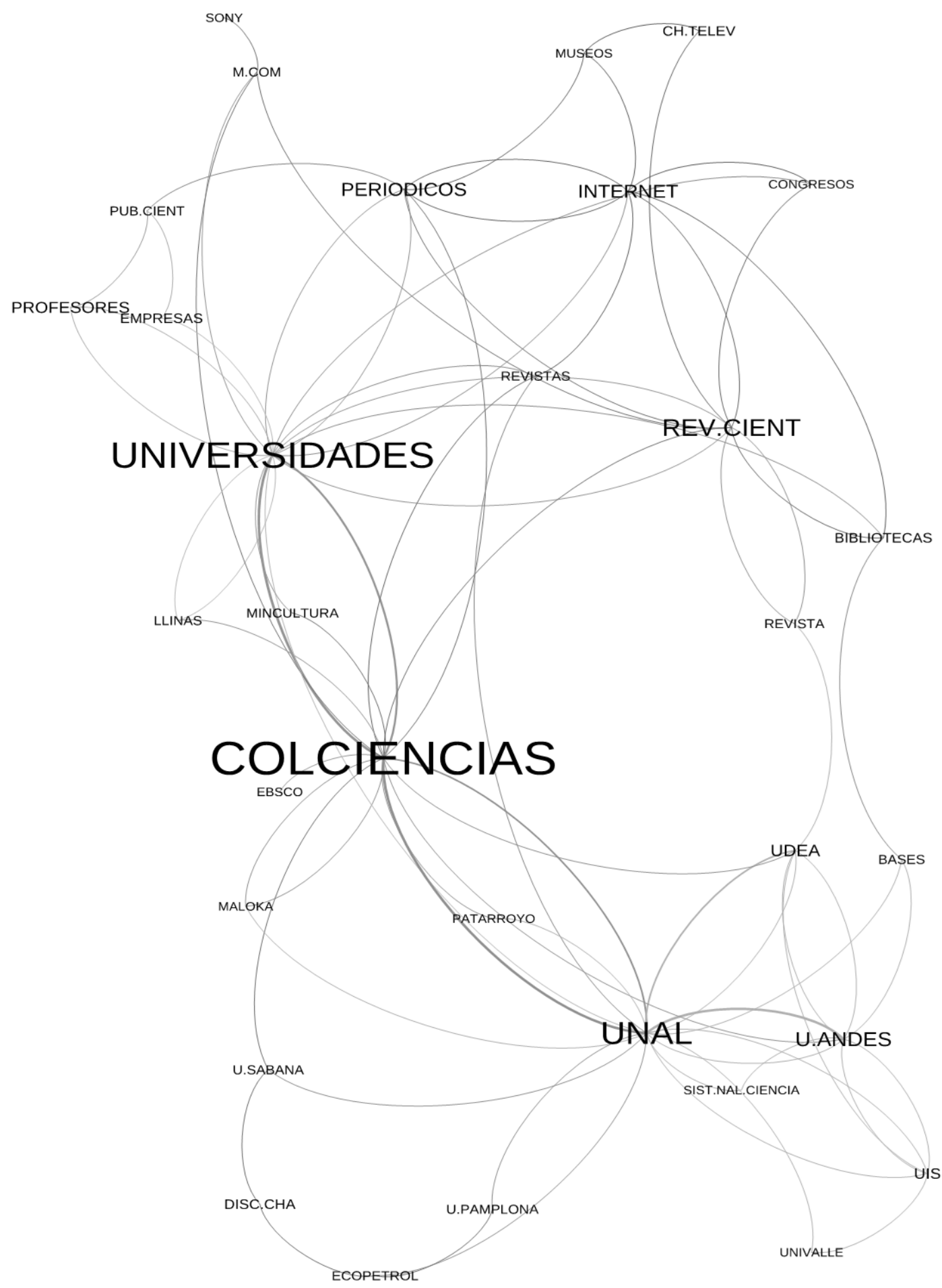

Figura 4. Análisis social de redes para identificar organizaciones, instituciones o personas divulgadoras de Ciencia y Tecnología en Colombia. Fuente: elaboración propia. 


\subsubsection{Dificultades para el desarrollo de la ciencia y la tecnología en Colombia}

Para el análisis de esta categoría se consideró clasificar a las dificultades en externas e internas a la ciencia y la tecnología. Para ello, se utilizó como referencia la cercanía o lejanía a la academia y a las capacidades de los encuestados. Dificultades asociadas cercanamente a la academia y a las capacidades encuestados se identificaron como internas, mientras que aquellas consideradas como alejadas a las dos variables anteriormente expuestas se determinaron como externas. En cuanto a las de orden externo, en orden descendente se identificó a la corrupción como la mayor de las dificultades $(72,6 \%)$, seguida por la falta de financiación $(71,4 \%)$, la falta de formación en investigación $(70,1 \%)$, el desconocimiento de la capacidad de la CyT para el desarrollo económico y social $(67,3 \%)$, la falta de voluntad política $(65,4 \%)$, la falta de redes entre la academia, la industria y el Estado (62,9 \%), la falta de instituciones que apoyen el desarrollo de la CyT $(61,3 \%)$ y la falta de apoyo de empresas privadas $(54,1 \%)$.

Por su parte, las dificultades internas fueron consideradas en menor medida por los participantes. En orden descendente: la baja calidad de la educación superior $(44,0 \%)$, la falta de patentes $(41,8 \%)$ y la baja cantidad de recursos humanos $(38,4 \%)$. En cuanto a este último ítem, al comparar por subgrupos de estudio, se identificó que los docentes/directivos (37,0\%), los estudiantes $(37,6 \%)$ y los profesionales graduados $(40,7 \%)$ lo ven como la menor de las dificultades, lo cual podría asociarse al optimismo que los encuestados tienen frente a las actividades académicas a las cuales se encuentran o encontraron vinculados. Por otro lado, cuando se analiza la falta de patentes como una dificultad para el desarrollo científico y tecnológico en Colombia, se observa que los profesionales graduados $(37,0 \%)$, los estudiantes $(42,9$ $\%)$ y los docentes y directivos $(48,1 \%)$ no la consideran como un aspecto relevante para que el desarrollo se pueda dar.

\subsection{Participación ciudadana}

En cuanto a la subcategoría de Aportes de la ingeniería química en la ciencia y la tecnología, para los encuestados la ingeniería química aporta a la CyT, principalmente, en la solución de problemáticas ambientales $(89,0 \%)$, el diseño, la invención y la fabricación de herramientas, componentes, procesos y sistemas para la industria química $(86,9 \%)$ y la generación de ideas creativas $(85,5 \%)$. En ese sentido, es importante notar que el sitio común entre el ingeniero químico colombiano y la participación ciudadana se da desde la solución o mitigación de los riesgos medioambientales que la industria química genera, lo cual es congruente con la tendencia actual de esta profesión hacia la "preocupación por el medioambiente y la seguridad" industrial [22] y de los grupos civiles de participación ciudadana frente a los riesgos asociados con la CyT [23]. Por su parte, en la categoría de Contribución de la ingeniería química en la resolución de problemáticas sociales, económicas y ambientales, los encuestados perciben que la mayor contribución de esta profesión consiste en la solución del problema de la contaminación ambiental $(95,6 \%)$, la gestión del recurso hídrico $(93,7 \%)$ y la solución a la sobreexplotación $(93,4$ $\%)$, lo cual concuerda con lo encontrado en apartados anteriores y en la cantidad de investigaciones y publicaciones asociadas con el tema [24] [25]. Finalmente, la contribución de esta disciplina en la solución de problemáticas sociales tales como la pobreza $(51,6 \%)$, las epidemias $(49,7 \%)$ o la solución del conflicto armado colombiano $(15,4 \%)$ son en las que, según los encuestados, menor incidencia tiene la IQ. Por tanto, se conserva la tendencia observada previamente, en la cual los currículos separan implícitamente las problemáticas ambientales de las sociales [26], de las cuales las primeras son las únicas en las que la IQ podría influir.

\section{Conclusiones}

El estudio de Exploración de la PSCyT de los ingenieros químicos colombianos y sus docentes se desarrolló desde los conceptos de RSCyT y participación ciudadana, bajo la hipótesis de que los ingenieros químicos tienen una percepción social similar a los resultados hallados en estudios nacionales o de otros grupos poblacionales. Asimismo, se utilizó la encuesta como método de recolección de los datos cuantitativos y cualitativos. Es decir, con este estudio se realizó una primera aproximación a la forma como este grupo poblacional percibe estos dos conceptos y cómo se percibe como actor científico y tecnológico desde un análisis general y otros segmentado según los subgrupos poblacionales (estudiantes, profesionales graduados, directivos y docentes). Con el fin de orientar la construcción de las conclusiones relacionadas con el objetivo general y la hipótesis propuesta, se ordenan los hallazgos en las cuatro temáticas que se estructuraron desde el Marco teórico (creencias e ideas, acciones, valores y participación ciudadana).

\subsection{Creencias e ideas}

En términos generales, la ciencia es considerada mayoritariamente desde la concepción neopositivista del conocimiento. Existen tendencias marcadas a asociarla con la investigación y un método que utilice la experimentación en el laboratorio para probar lo supuesto. De esta forma, la anterior afirmación se acerca 
al concepto del método científico como aquel método predominante para el desarrollo de la ciencia. También es claro que, para la mayoría de los encuestados, la ciencia produce conocimiento; es decir, que a pesar de que los encuestados no reconocen a la ciencia como un proceso social, cultural, económico o político que se encuentra mediado por las relaciones, sí muestran como resultado de esta al conocimiento. A su vez, esta es identificada, únicamente, como una actividad que genera nuevos conocimientos útiles para un desarrollo económico futuro (innovación), y no como un proceso sociohistóricamente situado que se encuentra permeado transversalmente con otros procesos. De esta forma, los encuestados identifican la ciencia desde una perspectiva neopositivista e instrumental que podría estar relacionada con el concepto heredado de la ciencia, el cual se encuentra integrado al estilo de educación impartido en la academia.

Por otro lado, la tecnología es vista por los encuestados desde un enfoque instrumentalista artefactual, la cual podría potenciar las posibilidades de un futuro mejor en términos económicos y que es muy cercana a las personas en su cotidianidad. Es decir, los encuestados tienen una actitud positiva hacia este proceso, lo cual podría estar mediado por la facilidad de acceso a la información y a los artefactos, dado el avance supuesto por la sociedad de la información. Asimismo, no se observan manifestaciones negativas o preventivas hacia la tecnología, lo cual permite suponer que esta es vista desde una visión neutral, sin cargas de las intenciones desde la que fue elaborada, y no desde una visión en la que se incluyan los factores políticos, sociales, culturales o económicos asociados a esta actividad. Igualmente, esta concepción de tecnología se enfoca desde la concepción intelectualista de la tecnología (tecnología como producto de la ciencia), lo cual la relaciona como un tipo de ciencia aplicada. De tal manera, las concepciones intelectualista y artefactual de la tecnología que se observan en este estudio exploratorio se asemejan y acotan a la definición restringida de tecnología de Pacey.

Por su parte, la producción y divulgación científica y tecnológica están visibilizadas desde la perspectiva de que ciertas instituciones (universidades, centros de investigación o el Departamento Administrativo de Ciencia, Tecnología e Innovación, Colciencias), científicos reconocidos (médicos colombianos, principalmente) o las redes sociales son quienes realmente influyen en estos procesos. Igualmente, la gran mayoría de los encuestados identificaron a Colciencias como institución productora de $\mathrm{CyT}$ en el país, a sabiendas que es el organismo gestor de la CyT bajo el marco de Sistema Nacional de Ciencia, Tecnología e Innovación, más no un organismo que se enfoca directamente en la producción científica y tecnológica. Por otro lado, frente a la divulgación científica y tecnológica, es notoria la relación entre la edad de los encuestados y su actividad -estudiante, graduado o docente/directivo-, lo cual mostró cómo es la percepción de los encuestados según el grupo al que pertenecen. Así, por ejemplo, al tener una edad mayor y ser docente o directivo se concibe la divulgación escrita en revistas especializadas como una forma importante de divulgación científica y tecnológica, mientras que al ser menor y estudiante las redes sociales son la forma más importante para divulgar la CyT.

\subsection{Acciones}

El internet, especialmente a través de las redes sociales, se visibiliza cómo el medio de comunicación más usado para consultar/recibir e informar/comunicar información de CyT, lo que podría asociarse a su facilidad de acceso y uso. No obstante, este fenómeno se observa con más frecuencia en grupos de menor edad (estudiantes, principalmente), quienes, posiblemente, han crecido más cercanamente a la tecnología utilizada con este fin (computadoras, teléfonos celulares inteligentes, tabletas, etc.). Sin embargo, el uso de esta no necesariamente corresponde a que haya una recepción o comunicación de información científica y tecnológica de calidad, según los parámetros aceptados por la comunidad científica.

Por otro lado, las revistas especializadas siguen siendo consideradas un medio de comunicación importante para informar sobre CyT, principalmente para los docentes y directivos (quienes son cercanos a la academia y, según los resultados, mayores en edad que el resto de los encuestados). Lo anterior podría asociarse a la historia y los acuerdos sociales en torno a la "correcta" comunicación de la ciencia, los cuales han considerado a este medio como única forma viable de comunicar conocimientos de calidad sobre CyT. Sin embargo, lo más interesante es que más de la mitad de los encuestados $(51,9 \%)$ no manifiestan publicar información en CyT, lo que podría visibilizar que la información que se comunica muy posiblemente no cumple con los requisitos de calidad implantados por la comunidad científica o que los encuestados no se encuentran vinculados activamente en actividades de investigación, cuyo resultado es publicar. Así, la información científica y tecnológica que se transfiere podría ser más cercana a la divulgación y no a la apropiación del conocimiento.

Por su parte, en términos generales se observó que los encuestados de la presente investigación manifiestan asistir en mayor medida a bibliotecas, museos de CyT, conferencias y semanas de la ciencia que los encuestados de otras investigaciones similares. Inicialmente, este fenómeno podría estar relacionado con la cercanía de los 
encuestados a la CyT, dada la disciplina con la que se encuentran relacionados. No obstante, al analizar por subgrupos poblacionales, los estudiantes y docentes/directivos son quienes más afirman acudir a estos espacios socialmente considerados como cercanos a la CyT, en comparación con los ingenieros químicos graduados. En ese orden de ideas, existe una posible relación directamente proporcional entre pertenecer a una IES, ya sea como estudiante, docente o directivo, y asistir a una actividad de ciencia y tecnología.

\subsection{Valores}

En términos generales, el interés de los encuestados frente a la ciencia y la tecnología se asocia con aquello que, al parecer, se concibe que hace la ingeniería química: productos y procesos industriales, su relación con las ciencias naturales básicas y la aplicación de la ciencia. Asimismo, estos muestran tener poco interés en el uso de herramientas electrónicas, uso de TIC, construcción de cosas, generar ideas de carácter técnico o enfocarse en problemas sociales. No obstante, a pesar de los valores obtenidos, el Gobierno Nacional no tiene dentro de sus prioridades la inversión en investigación en producción industrial, según los datos recogidos por el Observatorio Colombiano de Ciencia y Tecnología, lo cual podría traducirse en una posible falta de desarrollo económico asociado a este tipo de actividades y a la pérdida futura del interés por parte de los encuestados. Por tanto, de todo lo anterior se podría concluir que los intereses de los encuestados se encuentran más cercanos en aquellos atributos que son próximos al imaginario del quehacer del ingeniero (aplicación de la ciencia, ciencias básicas) o del ingeniero químico (diseño de productos, generación de nuevos procesos industriales, inventar y probar cosas), sin que necesariamente haya correspondencia con los intereses del Gobierno Nacional, y se encuentran alejados de la generación de ideas técnicas, construir cosas, resolver problemáticas sociales, en TIC o robots.

Frente a la importancia de la CyT, esta es muy similar a aquello que se considera que hace la ingeniería química y a su asociación con el desarrollo industrial y del sistema económico actual. De esta forma, se observa que los encuestados las consideran importantes para la innovación en las empresas, la utilización adecuada de los recursos naturales, el desarrollo económico nacional, el conocimiento y la preservación del entorno y el medioambiente, el cuidado de la salud y la prevención de enfermedades, la comprensión del mundo, la predicción y control de procesos, la generación de empleo y la toma de decisiones como consumidor. Es decir, la CyT son percibidas de forma positiva por los encuestados al igual que lo observado en la sección de Creencias e ideas. Por otro lado, la CyT no son percibidas como importantes para mejorar la toma de decisiones personales, relacionarse con los otros o construir opiniones políticas, lo cual podría confirmar que los tipos de CyT identificados están concebidos desde la concepción académica de la ingeniería química y sus áreas de influencia directa (industria y economía, p. ej.).

En cuanto a los usos más importantes de la CyT, se consideran aquellos que permiten hacer cambios en la sociedad o en las habilidades que se pueden adquirir. De esta forma, ocupa mayor relevancia cómo estos procesos ayudan a entender el entorno, conocer la verdad o solucionar problemas sociales (necesidades $\mathrm{o}$ expectativas altruistas), contrario a ganar dinero o tener poder (necesidades o expectativas personales). Esta es una tendencia muy similar a la de los resultados obtenidos en la III Encuesta nacional de percepción pública de la ciencia y la tecnología, desarrollada en Colombia [7], y en el documento del Proyecto iberoamericano de indicadores de percepción pública, cultura científica y participación ciudadana [6]. No obstante, en ciertos subgrupos poblacionales cambian estas percepciones, lo cual puede estar mediado por su acercamiento a la investigación (docentes y directivos) o por no aplicar a la ciencia y la tecnología en los terrenos laborales o investigativos (estudiantes).

Por otro lado, al valorar a la CyT desde la perspectiva de las dificultades que tienen estas para ser desarrolladas en el país, se observó que los encuestados manifiestan que la mayoría de estas se encuentran fuera de la academia y que se relacionan con situaciones políticas y económicas "ajenas". De tal forma, basado en la conclusión de que los encuestados consideran a la CyT como procesos individuales mediados por el conocimiento adquirido a través del método científico y de los científicos dentro de los laboratorios, se identificaron las siguientes conclusiones en cuanto a sus dificultades:

1. Los factores políticos y económicos son las barreras más grandes para el desarrollo científico y tecnológico, ya que son factores que no se pueden controlar desde la CyT. No obstante, esta afirmación mostraría que la ciencia y la tecnología no solo son agentes individuales que se encuentran mediados por factores internos. Es decir, los encuestados consideran que la generación de nuevo conocimiento y la capacidad técnica son labores de la ciencia y la tecnología, mientras que los recursos y la incidencia son responsabilidad de actores políticos que, al parecer, no entienden lo que sucede dentro de ellas. De tal forma, los encuestados mencionan implícitamente la influencia de los factores externos a la CyT como parte de la labor propia e indispensable para el desarrollo de estas actividades.

2. Para los encuestados, las características asociadas a la academia, tales como la baja calidad de la 
educación superior (ES), la falta de patentes o el poco capital humano preparado para hacer CyT en el país no son consideradas como factores relevantes de incidencia en el desarrollo científico y tecnológico. Por tanto, los encuestados corroboran que, para el adecuado desarrollo de la CyT, se requiere en mayor medida acciones políticas o económicas, asociadas a acciones de mejoramiento del capital humano para la investigación o la mejora de la calidad de la educación superior, que permitan el desarrollo fluido de las actividades científicas y tecnológicas. A su vez, se observó que los encuestados manifiestan ser optimistas frente a las labores académicas y las capacidades investigativas del capital humano, lo cual podría evidenciar un sesgo optimista que no permite reflexionar sobre las dificultades internas en la academia y las potencialidades por explorar.

\subsection{Participación ciudadana}

Desde las posturas de lo que aporta la IQ a la ciencia y la tecnología y su contribución a la resolución de problemáticas sociales, económicas y ambientales, se encontró que los encuestados tienden a considerar que la IQ aporta en la solución de problemáticas ambientales y en el diseño, invención y fabricación de herramientas, componentes, procesos y sistemas para la industria química y generación de ideas creativas e innovadoras, mas no en la solución de problemáticas sociales. De tal forma, los estudiantes, profesionales graduados $\mathrm{y}$ docentes/directivos de ingeniería química encuestados encuentran como punto común de interés la solución de problemáticas ambientales por medio de herramientas, máquinas, etc., las cuales se crean a partir de ideas creativas e innovadoras. Lo anterior da respuesta a lo establecido en el enfoque de protección del medioambiente, visto desde los currículos de las universidades y de los riesgos de contaminación ambiental que puede ocasionar la industria química. Por tanto, se observa que un posible nodo de agrupación civil de los ingenieros químicos estaría en la resolución, control y gestión de los riesgos y problemáticas ambientales. Por otra parte, las problemáticas sociales no son consideradas como un área en la que la IQ aporte. No obstante, esa afirmación es contradictoria con lo expresado en lo anteriormente descrito, debido a que los encuestados implícitamente están separando a las problemáticas sociales de lo que suceda con el medioambiente, lo cual hace suponer que ellos se considerarán como agentes que intervienen, mas no pertenecen, al medioambiente.

De igual manera, al observar al ingeniero químico como un agente social que contribuye a la ciencia y la tecnología, se identificó que la mayoría de los encuestados manifiestan que ellos influencian a la CyT por medio de la solución de problemáticas ambientales, a través del diseño e invención de herramientas, maquinarias, etc., elaboradas a partir de ideas creativas e innovadoras, mas no en la solución de problemáticas sociales. Es decir, los encuestados enfocan su mirada en los problemas ambientales producidos por la industria química, tal y como ha sido inculcado desde la academia y como respuesta a la tendencia mundial a la conservación del medioambiente. En resumen, ya sea como interés para agruparse socialmente o como materia de reflexión, análisis y solución, las problemáticas ambientales son el punto de inflexión para la participación de esta población, mientras que las problemáticas sociales se plantean como ajenas o de poco interés al campo de acción de la IQ.

En la Figura 5 se encuentran esquematizadas las RSCyT, anteriormente descritas, a través de la identificación del núcleo y de la periferia de cada una de ellas, según lo considerado por Abric [4]. Igualmente, en las Figuras 6 y 7 se muestran las relaciones halladas entre creencias $e$ ideas y acciones, y entre valores y participación ciudadana, respectivamente, y los conceptos más relevantes para cada una de estas variables. Como conclusión de esto se logró identificar que la hipótesis de trabajo en la que se consideraba que los ingenieros químicos, al ser parte de la sociedad no profesional en ingeniería química, tienen una percepción social similar a los resultados hallados en estudios nacionales o de otros grupos poblacionales -tales como ciudadanos colombianos [7], ciudadanos de Iberoamérica [6] o estudiantes iberoamericanos [8]-, se consideraría verdadera en cuanto a las creencias e ideas sobre los conceptos de ciencia, tecnología y comunicación y consulta de temas científicos y tecnológicos; y falsa con relación a la asistencia a actividades de CyT, sobre cómo las valoran y las formas de participación como ciudadanos, lo cual muestra que el conocimiento obtenido desde la academia media estos factores. Por otra parte, debido a que este fue un estudio exploratorio de la PSCyT en ingenieros químicos, se considera que aún se podría profundizar en ciertos aspectos que no fueron tratados a profundidad:

1. El significado, localización y acciones que se relacionan con la producción científica y tecnológica para los ingenieros químicos colombianos, al igual que el papel que tiene Colciencias dentro de esta percepción.

2. El papel de las IES y los currículos en las concepciones particulares e intereses sobre la ciencia y la tecnología en los ingenieros químicos colombianos.

3. El papel de los ingenieros químicos colombianos en los procesos de divulgación y producción científica y tecnológica en el país y el tipo de información científica y tecnológica que divulgan a través de internet, específicamente a través de las redes sociales. 


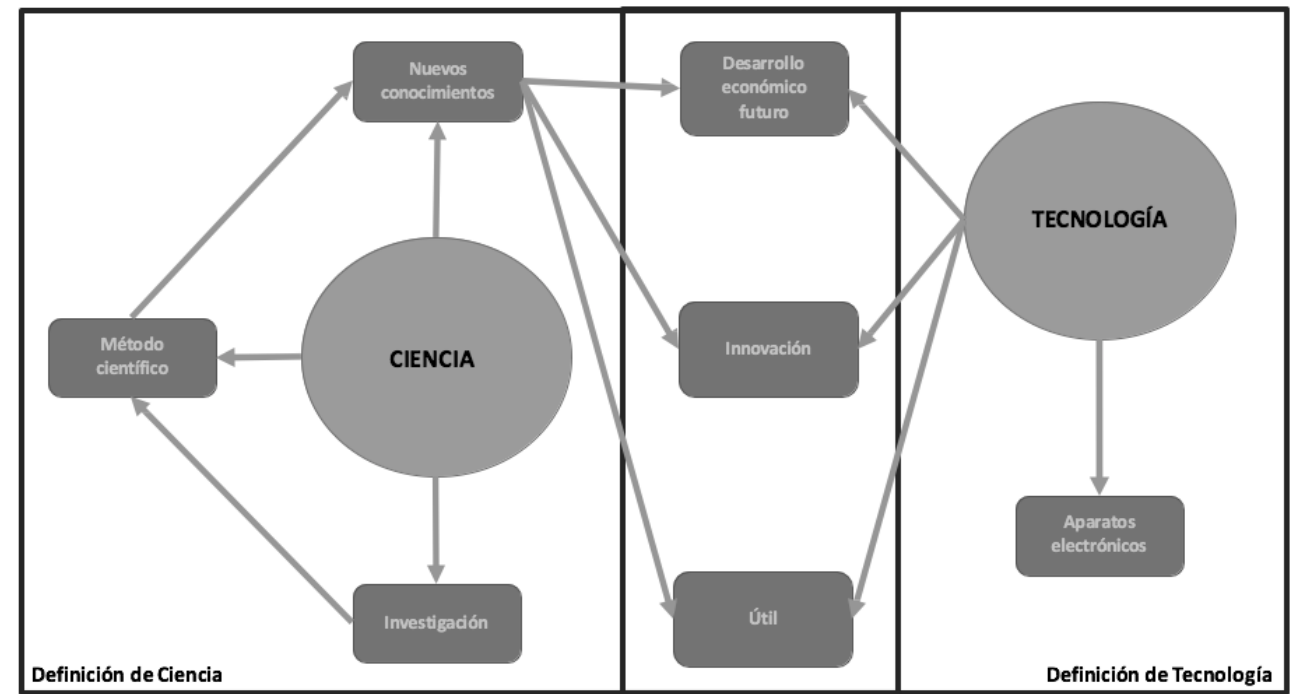

Figura 5. Representaciones sociales de la Ciencia y la Tecnología, según los ingenieros químicos colombianos.

Fuente: elaboración propia.

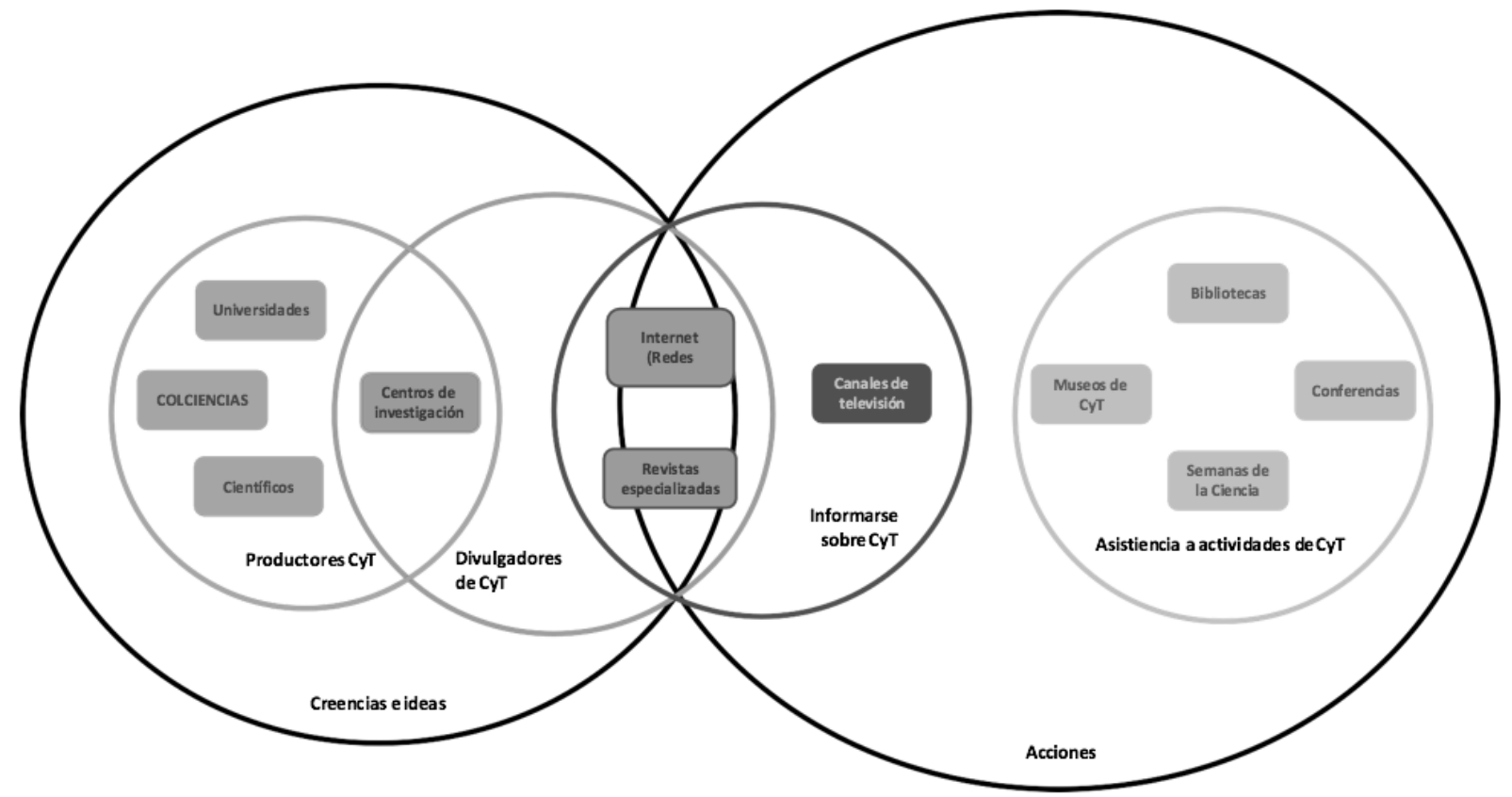

Figura 6. Relaciones halladas entre Creencias e Ideas y Acciones. Fuente: elaboración propia.

4. Los procesos de apropiación y cambios de actitud en caso de que se den- sobre la información científica y tecnológica divulgada por los ingenieros químicos a través de los diferentes medios.

5. El impacto de la información divulgada dentro de una posible sociedad del conocimiento en el interior de la ingeniería química.

6. El impacto de la vinculación laboral -tipo de experiencia, tiempo trabajando, en qué tipo de empresas, etc.- en la PSCyT de los ingenieros químicos colombianos.

7. En cuanto a los nodos de participación ciudadana, es importante indagar cuál es la concepción de medioambiente que tienen los ingenieros químicos y cuál es papel que le atribuyen a la sociedad frente a este tema. 8. Los impactos sociales, políticos y económicos de la ingeniería química, así como el impacto de esta disciplina en la ciencia y la tecnología. 


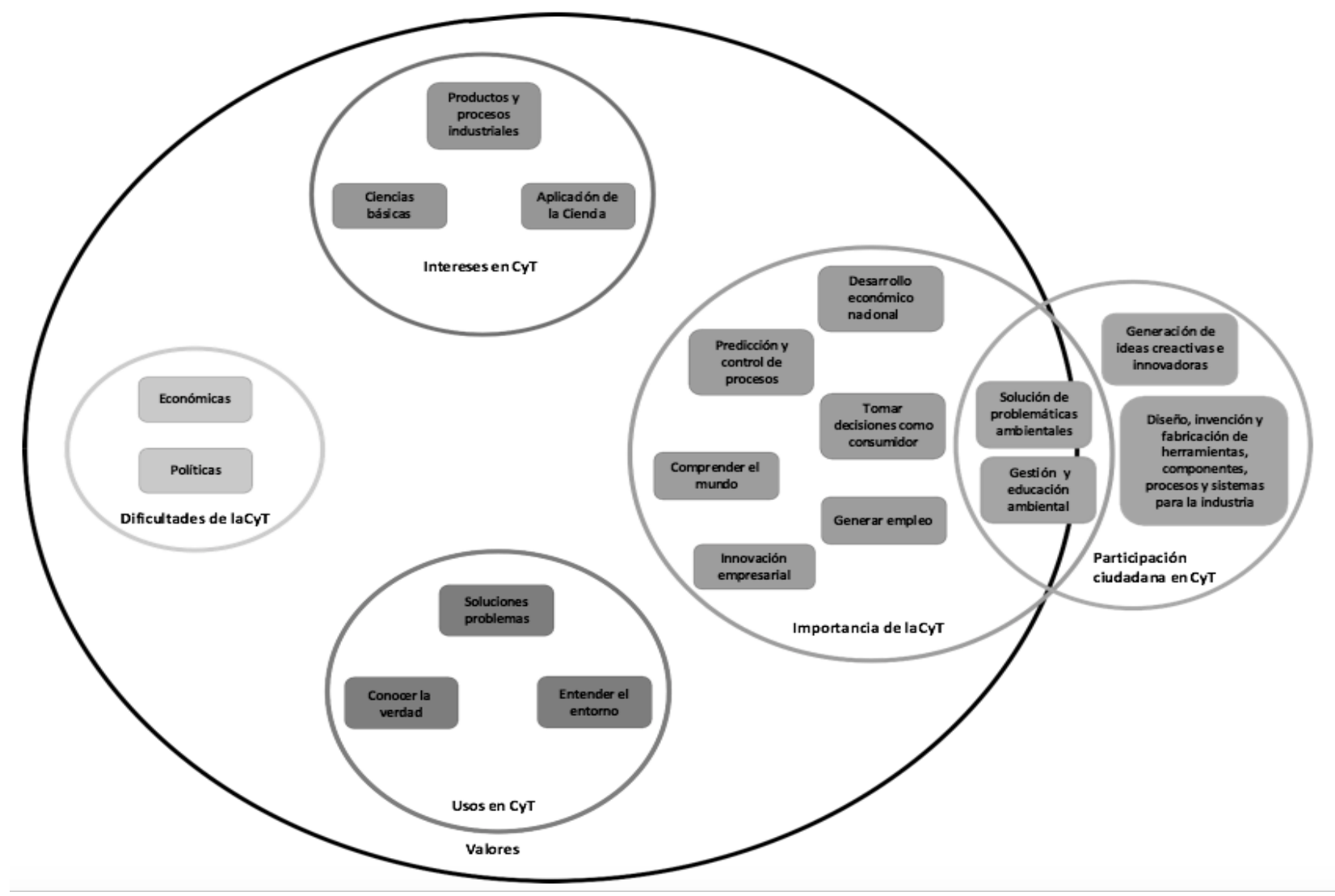

Figura 7. Relaciones halladas entre Valores y Participación Ciudadana. Fuente: elaboración propia.

9. Formulación de indicadores, planteados en el Manual de Antigua [27], para la elaboración de indicadores para encuestas de percepción pública de la ciencia y la tecnología, con población de las distintas ingenierías en Colombia y otros países, dada la naturaleza de estas profesiones y su acercamiento a la ciencia y la tecnología.

10. Oportunidad para que las IES, bibliotecas, entidades territoriales y museos de CyT generen proyectos que permitan aumentar la asistencia a estos centros, incluso después de graduarse de la universidad, en los ingenieros químicos graduados, y a mantener el interés de asistencia en los estudiantes, docentes y directivos.

Finalmente, esta exploración de la percepción social de la ciencia y la tecnología de estudiantes, profesionales graduados y docentes/directivos de ingeniería química es una oportunidad, en estudios futuros, para comprender cómo son y dónde se dan los mecanismos de construcción de este tipo de percepciones, identificar el papel de las universidades en estos procesos y reconocer formas alternativas de educación que permitan apropiar, por parte de los ingenieros químicos, una CyT más allá del laboratorio, la "verdad", los aparatos y las maquinarias.

\section{Referencias}

[1] F. D. Roosevelt, "Carta Roosevelt a Bush.pdf". Washington D.C., 1944.

[2] R. Hommes y C. M. Umaña, "Colombia en los próximos veinte años. El país que queremos", Departamento Nacional de Planeación, vol. 280, pp. 136, 2005.

[3] S. Moscovici, El psicoanálisis, su imagen y su público. Buenos Aires, 1979.

[4] J. Abric, Prácticas sociales y representaciones. México D.F., 2001.

[5] J. Durant, "What is scientific literacy?," Eur. Rev., vol. 2, no. 01, pp. 83-89, 1994. doi: $10.1017 / \mathrm{S} 1062798700000922$.

[6] OEI y RICYT, "Proyecto Iberoamericano de indicadores de percepción pública, cultura científica y 
participación ciudadana", Rev. Iberoam. ciencia, Tecnol. Soc. e innovación, vol. enero-abri, no. 5, 2003 [En línea]. Disponible

en: https://www.oei.es/historico/revistactsi/numero5/docum entos1.htm

[7] S. Daza-Caicedo, M. Lozano-Borda, E. M. Bueno Castellanos, Y. J. Gómez-Morales, M. Salazar Acosta, A. Jaime, J. P. Aguirre Guzmán, R. Rueda Ortiz, M. FrancoAve, y C. Osorio Marulanda, Percepciones de las ciencias y las tecnologías en Colombia. Resultados de la III Encuesta Nacional de Percepción Pública de la Ciencia y la Tecnología. Bogotá, 2014.

[8] C. Polino, Los estudiantes y la ciencia: encuesta a jóvenes iberoamericanos. Buenos Aires, 2011.

[9] C. E. Vargas-Ordoñez, "Exploración de la percepción social de la ciencia y la tecnología de ingenieros químicos colombianos y sus docentes", Universidad Nacional de Quilmes, 2016.

[10] T. J. Fontalvo Herrera, J. Morelos Gómes, y A. Mendoza Mendoza, "Incidencia de la certificación ISO 9001 en los indicadores de productividad y rentabilidad en empresas de zona franca-Barranquilla mediante análisis discriminante", Rev. UIS Ing., vol. 11, no. 2, pp. 215-225, 2012.

[11] E. García, J. C. González, J. A. López-Cerezo, J. L. Luján, M. Martín, C. Osorio, y C. Valdés, Ciencia, Tecnología y Sociedad: una aproximación conceptual. Madrid, 2001.

[12] R. J. Gómez, "Progreso, determinismo y pesimismo tecnológico", Rev. Redes, vol. 4, no. 10, pp. 59-94, 1997.

[13] A. H. G. Panqueva, "Ambientes virtuales para participar en la sociedad del conocimiento", Informática Educ., vol. 11, no. 2, pp. 247-260, 1998.

[14] A. Pacey, The Culture of Technology. MIT press, 1983.

[15] J. M. Sánchez-Torres, M. P. González-Zabala, y M. P. Sánchez-Muñoz, "La Sociedad de la Información: Génesis, Iniciativas, Concepto y su Relación con las TIC", Rev. UIS Ing., vol. 11, no. 1, pp. 113-128, 2012.

[16] E. Pineda Ballesteros, A. R. Lizcano Dallos, y R. N. Lízcano Reyes, "Redes sociales y representación de conocimiento como apoyo a los procesos de aprendizaje", Rev. UIS Ing., vol. 11, no. 2, pp. 237-251, 2012.

[17] COSCE, "La ciencia en la TV, la actividad editorial e internet”, Com. Reflexión y Estud. la Cienc. en España, vol. 1, no. 1, pp. 148-157, 2011.

[18] R. Manager y N. Iso, "Introducción a la Información científica y técnica. Curso 2001-2002 Profesora asociada: Lourdes Castillo 1", Knowl. Creat. Diffus. Util., pp. 1-20, 2002.

[19] A. V. Giraldo, G. Asdrúbal Valencia, y A. Valencia Giraldo, "La relación entre la ingeniería y la ciencia", Rev. Fac. Ing. Univ. Antioquia, vol. junio, núm. 31, pp. 156-174, 2004.

[20] J. Sabato y N. Botana, "América Latina: Ciencia y tecnología en el desarrollo de la sociedad", en Tiempo latinomericano, Universitaria, Ed. Santiado de Chile, 1970.

[21] Á. Vázquez, J. A. Acevedo, y M. A. Manassero, "Papel de la educación CTS en una alfabetización científica y tecnológica para todas las personas", REEC Rev. electrónica enseñanza las ciencias, vol. 2, no. 2, pp. 80-111, 2003.

[22] Unesco, Engineering: Issues Challenges and Opportunities for Development. 2010.

[23] N. Invernizzi, "Participación Ciudadana en Ciencia y Tecnología. Algunas Reflexiones sobre el Papel de la Universidad Pública", Alteridades, vol. 15, no. 29, pp. 37-44, 2005.

[24] L. Plata, D. Rivera, L. Castro, C. Guzmán, y H. Escalante, "Jerarquización de tecnologías para el aprovechamiento industrial del subproducto de la digestión anaerobia del bagazo de fique", Rev. UIS Ing., vol. 11, núm. 2, pp. 171-185, 2011.

[25] C. Martínez-López, J. Torres-Agredo, y R. Mejíade-Gutiérrez, "Evaluación de la toxicidad de un residuo industrial estabilizado/solidificado con cemento portland", Rev. UIS Ing., vol. 13, núm. enero-junio, pp. 47-54, 2014.

[26] D. B. Maldonado, "Un punto de vista sobre la formación del ingeniero químico colombiano", Ing. $e$ Investig., vol. 3, núm. 4, pp. 53-61, 1985.

[27] C. Polino, Manual de Antigua. Indicadores de percepción pública de la ciencia y la tecnología. 2015. 